Editorial: Hospital Metropolitano

ISSN (impreso) 1390-2989 - ISSN (electrónico) 2737-6303

Edición: Vol. 28 No 4 (2020) octubre - diciembre

DOI: https://doi.org/10.47464/MetroCiencia/vol28/4/2020/52-59

URL: https://revistametrociencia.com.ec/index.php/revista/article/view/96

Pág: $52-59$

\title{
Identificación de microorganismos anaerobios reactivos a la prueba de Bana antes y después de la terapia periodontal no quirúrgica en pacientes diabéticos
}

\section{Identification of anaerobic microorganisms reactive to the Bana test before and after non-surgical periodontal therapy in diabetic patients}

\author{
Juana Alexandra Ayo Bedón' ${ }^{1}$, Mariela Cumandá Balseca Ibarra \\ Odontóloga por la Facultad de Odontología de la Universidad Central del Ecuador, Quito, Ecuador ${ }^{1}$ \\ PhD en Periodoncia; Especialista en Periodoncia; Doctora en Odontología; Coordinadora del \\ Posgrado de Periodoncia de la Facultad de Odontología de la Universidad Central del Ecuador, Quito, Ecuador ${ }^{2}$
}

Recibido: 05/10/2020 Aceptado: 15/10/2020 Públicado: 29/10/2020

\section{RESUMEN}

La prueba BANA determina la actividad proteolítica de las principales bacterias periodontales del complejo rojo, la facilidad y la versatilidad clínica de la prueba la ha convertido en una opción viable para valorar el tratamiento periodontal. El Objetivo de esta investigación es la identificación de microorganismos, anaerobios reactivos a la prueba BANA antes y después de la terapia periodontal no quirúrgica en pacientes diabéticos. Materiales y Métodos: para este estudio se trabajó con pacientes que acuden al Club de Diabéticos del Centro de Salud N.- 4 de Chimbacalle perteneciente al Ministerio de Salud Pública del Distrito Metropolitano de Quito. Se realizó un muestreo no probabilístico a conveniencia de un total de 30 pacientes diabéticos con edades de 35 a 75 años de edad de ambos sexos, a los cuales se les realizó el análisis microbiológico BANA, antes y después de la terapia periodontal no quirúrgica. Se realizó un estudio prospectivo antes de la terapia periodontal no quirúrgica, y un mes después en pacientes diabéticos con periodontitis crónica en sitios con profundidades de sondaje y nivel de inserción $\geq 5 \mathrm{~mm}$. Resultados: los resultados del estudio no mostraron diferencias significativas entre los pacientes del estudio antes y después de la terapia periodontal no quirúrgica para la prueba microbiológica BANA. Conclusiones: La terapia periodontal no quirúrgica es un tratamiento eficaz en la eliminación de la enfermedad periodontal siempre y cuando exista la colaboración del paciente. Los resultados de la prueba microbiológica en este estudio no demostraron diferencias significativas.

Palabras claves: Periodontitis, BANA, diabetes.

\section{ABSTRACT}

The BANA test determines the proteolytic activity of the main periodontal red complex bacteria, the ease and clinical versatility of the test has made it a viable option for assessing periodontal treatment. The objective of this research is the identification of anaerobic microorganisms reactive to the BANA test before and after non-surgical periodontal therapy in diabetic patients. Materials and Methods: For this study we worked with patients attending the Diabetic Club of the Health Center No. 4 of Chimbacalle belonging to the Ministry of Public Health of the Metropolitan District of Quito. A non-probabilistic convenience sampling of a total of 30 diabetic patients between 35 and 75 years of age of both sexes was carried out, who underwent BANA microbiological analysis before and after non-surgical periodontal therapy. A prospective study was performed before non-surgical periodontal therapy, and one month after in diabetic patients with chronic periodontitis in sites with probing depths and insertion level $\geq 5 \mathrm{~mm}$. Results: the results of the study showed no significant differences between study patients before and after non-surgical periodontal therapy for the microbiological BANA test.Conclusions: Non-surgical periodontal therapy is an effective treatment in the elimination of periodontal disease as long as there is patient cooperation. The results of the microbiological test in this study showed no significant differences.

Keywords: Periodontitis, BANA, diabetes.

\section{IDs Orcid}

https://orcid.org/0000-0002-8263-5178 https://orcid.org/0000-0002-7218-3622

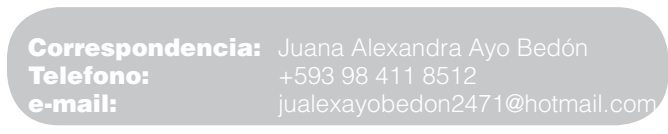




\section{INTRODUCCIÓN}

Dentro de las enfermedades infecciosas más comunes en cavidad bucal las afecciones periodontales sin duda se encuentran entre una de las más extendidas en distintas poblaciones a nivel mundial. La gravedad de la enfermedad periodontal puede estar influenciada por diferentes factores, como son las enfermedades sistémicas entre ellas la diabetes ${ }^{1}$.

Es indiscutible que la periodontitis y la diabetes asociadas pueden causar malestar en los pacientes, así como la calidad de vida de los individuos afectados, dentro del tratamiento de la periodontitis la terapia no quirúrgica que implementa hábitos de higiene oral, como la eliminación mecánica de los depósitos bacterianos se considera de primera elección². Si bien la mejoría de los pacientes con periodontitis ante la terapia periodontal es evidente y se ven beneficiados por el cambio en sus hábitos de higiene oral y otros beneficios como ausencia de sangrado gingival y halitosis, considerando que la respuesta al tratamiento puede variar en pacientes diabéticos ${ }^{3}$.

La diabetes y la enfermedad periodontal (EP) son dos condiciones estudiadas desde hace mucho tiempo debido a la gran relación de su etiopatogenia inflamatoria común, razón por la cual se considera a la enfermedad periodontal como la sexta complicación de la diabetes o viceversa ${ }^{4}$. Varios estudios han indicado las consecuencias que la diabetes mellitus (DM) ocasiona dentro de la cavidad oral en especial a nivel de los tejidos periodontales los cuales son alterados fisiológicamente, produciendo a larga data la pérdida de inserción periodontal por la concentración alta y de forma crónica de la glucosa en sangre, debido a la falta de control adecuado de la misma ${ }^{4}$. Estudios realizados han podido comprobar que en los pacientes con diabetes presentan un riesgo incrementado para el desarrollo de la enfermedad periodontal, estos antecedentes han permitido proponer que el mal control metabólico de la diabetes es un factor importante que puede modular negativamente la evolución de la enfermedad periodontal ${ }^{5}$. La prevención de las afecciones periodontales en el paciente diabético es el mejor tratamiento del que se dispone en la actualidad, el conocimiento y la actualización permanente de los aspectos fisiopatológicos en la correlación de ambas entidades es clave para seleccionar y ejecutar un temprano y adecuado tratamiento, no solo con el fin de reducir la morbilidad de la infección local, sino también de influir indirectamente en la salud general ${ }^{6}$. La evolución de la periodontitis es más rápida y avanzada en los pacientes diabéticos, estos pacientes presentan bolsas más profundas, más movilidad dentaria, más pérdida de inserción, menor nivel óseo y peor respuesta al tratamiento, haciendo que las consecuencias de la periodontitis se hagan más evidentes ${ }^{7,8}$.
Las bacterias más frecuentemente asociadas a las periodontitis son los anaerobios Gram-negativos, que usan principalmente proteínas y péptidos como fuente de energía. (Loesche et al. 1990). Así, una o más enzimas proteolíticas, exclusivas de tales bacterias, servirían de marcadores moleculares y consecuentemente como marcadores de riesgo y/o de la infección causada por estos microorganismos ${ }^{9}$.

Estos microorganismos para poder utilizar proteínas y péptidos como fuentes de energía forman enzimas capaces de degradarlas, pero algunos de ellos tienen una enzima semejante a la tripsina, capaz de hidrolizar sustratos que tengan arginina ligada a un cromóforo. El test más conocido ha sido el BANA que analiza del péptido sintético N-Benzoil-DL-Arginina-2-Naftilamida que sufre hidrolisis por la microbiota subgingival ${ }^{11-12}$. Éste fue el primer substrato sugerido con posible valor diagnóstico (Loesche 1986). El test es realizado colocando una muestra de placa dental en una tira de papel impregnada con el péptido BANA. Si hay por lo menos, uno de los tres patógenos Tannerella forsythia, Tannerella dentiola y Porphyromonas gingivalis, el BANA es hidrolizado promoviendo un cambio de color azul, y la intensidad de color corresponde a la cantidad de microorganismos ${ }^{12}$.

\section{MATERIALES Y METODOS}

Estudio observacional, analítico y de corte longitudinal, se realizó en pacientes con Diabetes tipo 2 este estudio se trabajó con pacientes que acuden al Club del Diabéticos del Centro de Salud Nº4 de Chimbacalle perteneciente al Ministerio de Salud Pública del Distrito Metropolitano de Quito, el estudio fue aprobado por el comité de ética de la Universidad Central del Ecuador.

\section{Escala de bana utilizado}

La escala de BANA nos indica la reacción, que puede determinar la habilidad de ciertos tipos de especies bacterianas de placa subgingival de hidrolizar la enzima lo que podría determinar su presencia correlacionando los niveles de la reacción enzimática con la proporción de patógenos presentes, dando una significancia diagnostica. Por lo tanto, se va a comparar la presencia de actividad enzimática bacteriana en pacientes diabéticos con periodontitis, y sin periodontitis por escalas.

Reacción negativa cuando no observamos coloración azul en la banda, sin embargo posee un rango de 1000-5000 UFC; débilmente positiva entre 10000 a 100000 UFC, y positiva mayor a 100000 UFC en el sitio que se tomó la muestra.

- Bana Negativo 0 = 1000-5000 UFC (Unidad formadora de colonias) 
- Bana Leve Positivo 1=10000-100000 UFC (Unidad formadora de colonias)

- Bana Positivo 2=Mayor a 100000UFC (Unidad formadora de colonias)

\section{Población de estudio y muestra}

Para este estudio se trabajó con pacientes que acuden al Club del Diabéticos del Centro de Salud N.-4 de Chimbacalle perteneciente al Ministerio de Salud Pública del Distrito Metropolitano de Quito .Se realizara un muestreo no probabilístico por conveniencia de un total de 30 pacientes, cuyo criterio de inclusión fue tener un rango de 35 a 75 años de edad y de ambos sexos, Mientras que el análisis la evaluación clínica será realizada por un observador entrenado y calibrado en la detección de enfermedad periodontal, el análisis microbiológico se llevará a cabo utilizando las pruebas BANA.

Se realizó el examen periodontal a cada uno de los pacientes, para ello se evaluaron todos los elementos dentales presentes en boca y se tomó una pieza dentaria determinada en cada uno de los pacientes evaluando la profundidad de sondaje en las cuatro superficies vestibular, lingual o palatino/mesial y distal de cada una de las piezas dentarias elegidas utilizando el sondeo periodontal como método de diagnóstico convencional, y la prueba BANA como método de diagnóstico no convencional. El criterio clínico para la determinación del área para la colección del material para el BANA fue la superficie con la bolsa más profunda de cada diente.

\section{Análisis estadístico}

Para el estudio estadístico, la información fue, codificada y archivada en un archivo de Excel y el paquete estadístico SPSS 22, se realizó estadística descriptiva. Para verificar la relación entre variables, se utilizó pruebas de normalidad de Anova, Chi cuadrado de Pearson y Kruskal Wallis.

\section{RESULTADOS}

Según las edades del personal evaluado, el $40 \%$ son menores de 60 años y el $60 \%$ son mayores o iguales a 60 años (tercera edad) con relacion al genero del grupo evaluado, el $40 \%$ son de genero masculino y el $60 \%$ son de genero femenino.

Tabla 1. Descripción de la muestra

\begin{tabular}{cccc} 
Variables & Indicador & Frecuencia & Porcentaje \\
\hline Edades & $\begin{array}{c}\text { Menor que } \\
60\end{array}$ & 12 & $40,0 \%$ \\
& $\begin{array}{c}\text { Mayor o } \\
\text { igual a } 60\end{array}$ & 18 & $60,0 \%$ \\
& & & \\
& &
\end{tabular}

\begin{tabular}{|c|c|c|c|}
\hline \multirow{3}{*}{ Genero } & Masculino & 12 & $40,0 \%$ \\
\hline & & & \\
\hline & Femenino & 18 & $60,0 \%$ \\
\hline \multirow{2}{*}{ Sangrado /Antes } & $\begin{array}{c}\text { Sin } \\
\text { Sangrado }\end{array}$ & 3 & $10,0 \%$ \\
\hline & $\begin{array}{c}\text { Con } \\
\text { Sangrado }\end{array}$ & 27 & $90,0 \%$ \\
\hline NI Antes & Positivo & 30 & $100,0 \%$ \\
\hline PS Antes & $\begin{array}{l}>5 \text { Bolsa } \\
\text { Periodontal }\end{array}$ & 30 & $100,0 \%$ \\
\hline \multirow{2}{*}{ Bana Antes } & $\begin{array}{l}\text { Leve } \\
\text { Positivo }\end{array}$ & 12 & $40,0 \%$ \\
\hline & Positivo & 18 & $60,0 \%$ \\
\hline \multirow{3}{*}{ PS Después } & $\leq 4 \mathrm{~mm}$ & 18 & $600 \%$ \\
\hline & & & \\
\hline & $\geq 4 \mathrm{~mm}$ & 12 & $40,0 \%$ \\
\hline \multirow[t]{2}{*}{ NI Después } & Positivo & 30 & $100,0 \%$ \\
\hline & Negativo & 10 & $33,3 \%$ \\
\hline \multirow[t]{2}{*}{ Bana Después } & $\begin{array}{c}\text { Leve } \\
\text { Positivo }\end{array}$ & 17 & $56,7 \%$ \\
\hline & Positivo & 3 & $\%$ \\
\hline \multirow{2}{*}{ Sangrado Después } & $\begin{array}{c}\text { Sin } \\
\text { Sangrado }\end{array}$ & 22 & $73,3 \%$ \\
\hline & $\begin{array}{c}\text { Con } \\
\text { Sangrado }\end{array}$ & 8 & $26,7 \%$ \\
\hline
\end{tabular}

\section{Antes del raspado periodontal}

- Sangrado antes: Sin sangrado tienen 3 evaluados que equivalen al 10,0\% y con sangrado tienen 27 que equivale al 90,0\%.

- NI. Antes: solo se tienen Positivo esto es 30 evaluados que corresponde al 100,0\%

- PS. Antes: solo se tienen $>5 \mathrm{~mm}$ Bolsa Periodontal esto es 30 evaluados y corresponde al $100,0 \%$

- Bana antes: se tienen LEVE POSITIVO con 12 evaluados que corresponde al $40,0 \%$ y POSITIVO con 18 evaluados y corresponde al 60,0\% 
Tabla 2. Modelos lineales Generalizados: Antes de la prueba BANA

\begin{tabular}{|c|c|c|c|c|c|c|c|c|c|c|}
\hline \multicolumn{11}{|c|}{ Estimaciones de parámetro } \\
\hline \multirow[b]{2}{*}{ Parámetro } & \multirow[b]{2}{*}{ B } & \multirow[b]{2}{*}{$\begin{array}{c}\text { Error } \\
\text { estándar }\end{array}$} & \multicolumn{2}{|c|}{$\begin{array}{c}95 \% \text { de intervalo } \\
\text { de confianza de } \\
\text { Wald }\end{array}$} & \multicolumn{3}{|c|}{ Contraste de hipótesis } & \multirow{2}{*}{$\operatorname{Exp}(B)$} & \multicolumn{2}{|c|}{$\begin{array}{c}95 \% \text { de intervalo de } \\
\text { confianza de Wald } \\
\text { para } \operatorname{Exp}(B)\end{array}$} \\
\hline & & & Inferior & Superior & $\begin{array}{c}\text { Chi-cuadrado } \\
\text { de Wald }\end{array}$ & gl & $\begin{array}{l}\text { Sig. } \\
(p=)\end{array}$ & & Inferior & Superior \\
\hline (Interceptación) & 0,5 & 0,2 & 0,1 & 1,0 & 5,8 & 1,0 & 0,016 & 1,715 & 1,1 & 2,7 \\
\hline Masculino & $-0,2$ & 0,3 & $-0,7$ & 0,4 & 0,2 & 1,0 & 0,620 & 0,860 & 0,5 & 1,6 \\
\hline Femenino & 0,0 & . & . & . & . & . & . & 1,000 & . & . \\
\hline Menor que 60 & $-0,1$ & 0,3 & $-0,7$ & 0,5 & 0,1 & 1,0 & 0,712 & 0,895 & 0,5 & 1,6 \\
\hline $\begin{array}{l}\text { Mayor o igual } \\
\text { a } 60\end{array}$ & 0,0 & . & . & . & . & . & . & 1,000 & . & . \\
\hline Sin sangrado & 0,3 & 0,4 & $-0,6$ & 1,2 & 0,4 & 1,0 & 0,521 & 1,330 & 0,6 & 3,2 \\
\hline Con sangrado & 0,0 & . & . & . & . & . & . & 1,000 & . & . \\
\hline (Escala) & 1,0 & & & & & & & & & \\
\hline
\end{tabular}

\section{Después del raspado periodontal}

- Sangrado después: Sin sangrado tienen 22 evaluados que equivalen al $73,3 \%$ y con sangrado tienen 8 evaluados que equivale al $26,7 \%$.

- NI. Después: solo se tienen Positivo esto es 30 evaluados que corresponde al 100,0\%
- PS Después: se tienen $<=4 \mathrm{~mm}$ salud con 18 evaluados que equivale al $60,0 \%$ y $>4 \mathrm{~mm}$ Bolsa Periodontal esto es 12 evaluados y corresponde al $40,0 \%$

- Bana después: se tienen 10 evaluados con NEGATIVO esto es el 33,3\%, LEVE POSITIVO con 17 evaluados que corresponde al $56,7 \%$ y $\mathrm{PO}$ SITIVO con 3 evaluados y corresponde al 10,0\%

Tabla 3. Modelos lineales generalizados; Después del BANA

\begin{tabular}{|c|c|c|c|c|c|c|c|c|c|c|}
\hline \multicolumn{11}{|c|}{ Estimaciones de parámetro } \\
\hline \multirow[b]{2}{*}{ Parámetro } & \multirow[b]{2}{*}{ B } & \multirow[b]{2}{*}{$\begin{array}{l}\text { Error } \\
\text { estándar }\end{array}$} & \multicolumn{2}{|c|}{$\begin{array}{c}95 \% \text { de intervalo } \\
\text { de confianza de } \\
\text { Wald }\end{array}$} & \multicolumn{3}{|c|}{ Contraste de hipótesis } & \multirow[t]{2}{*}{$\operatorname{Exp}(B)$} & \multicolumn{2}{|c|}{$\begin{array}{c}95 \% \text { de intervalo de } \\
\text { confianza de Wald } \\
\text { para } \operatorname{Exp(B)}\end{array}$} \\
\hline & & & Inferior & Superior & $\begin{array}{l}\text { Chi-cuadrado } \\
\text { de Wald }\end{array}$ & gl & $\begin{array}{l}\text { Sig. } \\
(p=)\end{array}$ & & Inferior & Superior \\
\hline (Interceptación) & 1,59 & 0,88 & $-0,13$ & 3,31 & 3,27 & 1,00 & 0,07 & 4,901 & 0,87 & 27,48 \\
\hline Masculino & $-1,25$ & 0,83 & $-2,87$ & 0,37 & 2,29 & 1,00 & 0,13 & 0,287 & 0,06 & 1,45 \\
\hline Femenino & 0,00 & & . & . & . & . & . & 1,000 & . & \\
\hline Menor que 60 & $-1,84$ & 0,78 & $-3,38$ & $-0,31$ & 5,54 & 1,00 & 0,02 & 0,158 & 0,03 & 0,74 \\
\hline $\begin{array}{l}\text { Mayor o igual } \\
\text { a } 60\end{array}$ & 0,00 & & . & . & & . & . & 1,000 & . & \\
\hline Sin sangrado & $-1,63$ & 0,79 & $-3,18$ & $-0,08$ & 4,22 & 1,00 & 0,04 & 0,197 & 0,04 & 0,93 \\
\hline $\begin{array}{l}\text { Con } \\
\text { sangrado }\end{array}$ & 0,00 & & . & . & . & . & . & 1,000 & . & . \\
\hline$<=4 \mathrm{~mm}$ : salud & 0,13 & 0,46 & $-0,77$ & 1,03 & 0,08 & 1,00 & 0,77 & 1,141 & 0,46 & 2,81 \\
\hline $\begin{array}{l}>4 \text { Bolsa } \\
\text { Periodontal }\end{array}$ & 0,00 & & . & & & . & & 1,000 & . & \\
\hline (Escala) & 1,00 & & & & & & & & & \\
\hline
\end{tabular}

En después según el modelo de Regresión de Poisson, las variables que son significativamente representativas son la edad y el sangrado. Las personas de género masculino tienen un $71,3 \%(1-0,287)$ menos de riesgo de tener BANA que los que son de género femenino. La variable no es representativa $(p>0,05) \quad$ Las personas Menores a 60 años tienen un $84,2 \%(1-0,158)$ menos de riesgo de tener BANA que los que son Mayores o iguales a 60 años. La variable si es representativa $(p<0,05)$ Las personas sin sangrado tienen un 80,3\% (1-0,197) menos de riesgo de tener BANA que los que están con sangrado. La variable si es representativa $(p<0,05)$. 
Tabla 4. Correlaciones no paramétricas (Antes y después contra el Bana)

\begin{tabular}{|c|c|c|c|c|}
\hline \multirow{2}{*}{ VARIABLES } & \multicolumn{4}{|c|}{ BANA } \\
\hline & Antes & Interpretación & Después & Interpretación \\
\hline Genero & 0,167 & Pequeña & $-0,040$ & Pequeña \\
\hline Edades & 0,167 & Pequeña & 0,707 & $\begin{array}{c}\text { Alta } \\
\text { Positiva }\end{array}$ \\
\hline Sangr/Ante & $-0,272$ & Pequeña & 0,044 & Pequeña \\
\hline $\begin{array}{c}\text { Sangr/ } \\
\text { Después }\end{array}$ & $-0,123$ & Pequeña & 0,572 & $\begin{array}{c}\text { Moderada } \\
\text { Positiva }\end{array}$ \\
\hline $\begin{array}{c}\text { Ps } \\
\text { Despues }\end{array}$ & 0,250 & Pequeña & $-0,018$ & Pequeña \\
\hline
\end{tabular}

Las correlaciones (Rho de Spearman) entre cada variable y el índice BANA ANTES son pequeñas. No es fuerte la relación.

Las correlaciones (Rho de Spearman) entre cada variable y el índice BANA DESPUÉS son alta positiva con las edades y moderada positiva con el sangrado. Es fuerte la relación, lo que se confirma con los valores de la regresión de Poisson.

Tabla 5. Variables con BANA antes del raspado Periodontal

\begin{tabular}{|c|c|c|c|c|c|c|c|c|}
\hline & & \multicolumn{4}{|c|}{ Bana Antes } & \multirow{2}{*}{\multicolumn{2}{|c|}{ Total }} & \multirow{3}{*}{$p=$} \\
\hline & & \multicolumn{2}{|c|}{ Leve Positivo } & \multicolumn{2}{|c|}{ Positivo } & & & \\
\hline & & Cant & $\%$ & Cant & $\%$ & Cant & $\%$ & \\
\hline \multirow{2}{*}{ Genero } & Masculino & 6 & $50,0 \%$ & 6 & $50,0 \%$ & 12 & $100,0 \%$ & \multirow{2}{*}{0,361} \\
\hline & Femenino & 6 & $33,3 \%$ & 12 & $66,7 \%$ & 18 & $100,0 \%$ & \\
\hline \multirow{2}{*}{$\begin{array}{l}\text { SANGR/ } \\
\text { antes }\end{array}$} & Sin Sangrado & 0 & $0,0 \%$ & 3 & $100,0 \%$ & 3 & $100,0 \%$ & \multirow{2}{*}{0,136} \\
\hline & Con Sangrado & 12 & $44,4 \%$ & 15 & $55,6 \%$ & 27 & $100,0 \%$ & \\
\hline $\mathrm{NI}$ antes & Positivo & 12 & $40,0 \%$ & 18 & $60,0 \%$ & 30 & $100,0 \%$ & - \\
\hline PS antes & > 5 Bolsa Periodontal & 12 & $40,0 \%$ & 18 & $60,0 \%$ & 30 & $100,0 \%$ & - \\
\hline \multirow{2}{*}{ Edades } & Menor que 60 & 6 & $50,0 \%$ & 6 & $50,0 \%$ & 12 & $100,0 \%$ & \multirow{2}{*}{0,361} \\
\hline & Mayor o igual a 60 & 6 & $33,3 \%$ & 12 & $66,7 \%$ & 18 & $100,0 \%$ & \\
\hline
\end{tabular}

Dividiendo para las filas, todos los valores de significación $(p=)$ son mayores a 0,05 , luego no se tienen diferencias significativas entre los porcentajes del BANA con relación a las variables.

\section{Genero}

- MASCULINO: El 50\% tienen BANA leve positivo (poca cantidad de colonias bacterianas) y el $50 \%$ tienen BANA positivo. (mayor cantidad de colonias bacterianas).

- FEMENINO: El 33,3\% tienen BANA leve positivo y el $66,7 \%$ tienen BANA positivo.

\section{Sangrado}

- SIN SANGRADO: El 0,0\% tienen BANA leve positivo y el $100 \%$ tienen BANA positivo.

- CON SANGRADO: El 44,4\% tienen BANA leve positivo y el $55,6 \%$ tienen BANA positivo.

\section{NI Antes}

- Positivo: El 40\% tienen BANA leve positivo y el $60 \%$ tienen BANA positivo.

\section{PS Antes}

- 5 bolsa Periodontal: El 40\% tienen BANA leve positivo y el $60 \%$ tienen BANA positivo.

\section{Edades}

- Menor que 60: El 50\% tienen BANA leve positivo y el $50 \%$ tienen BANA positivo

- Mayor o igual a 60: El 33,3\% tienen BANA leve positivo y el $66,7 \%$ tienen BANA positivo.

Dividiendo para las filas, algunos de los valores de significación $(p=)$ son menores a 0,05 , luego Si se tienen diferencias significativas entre los porcentajes del BANA con relación a las variables relacionadas. 
Tabla 6. Variables con BANA después del raspado radicular

\begin{tabular}{|c|c|c|c|c|c|c|c|c|c|c|}
\hline \multicolumn{11}{|c|}{ Bana despues } \\
\hline & & \multicolumn{2}{|c|}{ Leve Positivo } & \multicolumn{2}{|c|}{ Positivo } & \multicolumn{2}{|c|}{ POSITIVO } & \multicolumn{2}{|c|}{ Total } & \multirow{2}{*}{$p=$} \\
\hline & & Cant & $\%$ & Cant & $\%$ & Cant & $\%$ & Cant & $\%$ & \\
\hline \multirow{2}{*}{ Genero } & Masculino & 5 & $41,7 \%$ & 4 & $33,3 \%$ & 3 & $25,0 \%$ & 12 & $100,0 \%$ & \multirow{2}{*}{0,033} \\
\hline & Femenino & 5 & $27,8 \%$ & 13 & $72,2 \%$ & 0 & $0,0 \%$ & 18 & $100,0 \%$ & \\
\hline \multirow{2}{*}{$\begin{array}{l}\text { PS } \\
\text { después }\end{array}$} & $<=4 \mathrm{Mm}$ & 6 & $33,3 \%$ & 10 & $55,6 \%$ & 2 & $11,1 \%$ & 18 & $100,0 \%$ & \multirow{2}{*}{0,968} \\
\hline & $>4 \mathrm{Mm}$ & 4 & $33,3 \%$ & 7 & $58,3 \%$ & 1 & $8,3 \%$ & 12 & $100,0 \%$ & \\
\hline $\begin{array}{l}\text { NI } \\
\text { despues }\end{array}$ & Positivo & 10 & $33,3 \%$ & 17 & $56,7 \%$ & 3 & $10,0 \%$ & 30 & $100,0 \%$ & - \\
\hline \multirow{2}{*}{$\begin{array}{l}\text { SANGR/ } \\
\text { después }\end{array}$} & Sin Sangrado & 10 & $45,5 \%$ & 12 & $54,5 \%$ & 0 & $0,0 \%$ & 22 & $100,0 \%$ & \multirow{2}{*}{0,003} \\
\hline & Con Sangrado & 0 & $0,0 \%$ & 5 & $62,5 \%$ & 3 & $37,5 \%$ & 8 & $100,0 \%$ & \\
\hline \multirow{2}{*}{ Edades } & Menor que 60 & 9 & $75,0 \%$ & 3 & $25,0 \%$ & 0 & $0,0 \%$ & 12 & $100,0 \%$ & \multirow{2}{*}{0,000} \\
\hline & Mayor o igual a 60 & 1 & $5,6 \%$ & 14 & $77,8 \%$ & 3 & $16,7 \%$ & 18 & $100,0 \%$ & \\
\hline
\end{tabular}

\section{Genero (diferencias por genero $\mathrm{p}<0,05$ )}

- MASCULINO: El 41,7\% tienen BANA Negativo, el $33,3 \%$ tienen BANA leve positivo y el $25,0 \%$ tienen BANA positivo.

- FEMENINO: El 27,8\% tienen BANA Negativo, el $72,2 \%$ tienen BANA leve positivo y el $0,0 \%$ tienen BANA positivo.

\section{PS después}

- $\quad$ s4 mm: El 33,3\% tienen BANA Negativo, el
$55,6 \%$ tienen BANA leve positivo y el 11,1\% tienen BANA positivo.

- $>4$ mm: El 33,3\% tienen BANA Negativo, el $58,3 \%$ tienen BANA leve positivo y el $8,3 \%$ tienen BANA positivo.

\section{NI después}

Positivo: El 33,3\% tienen BANA Negativo, el 56,7\% tienen BANA leve positivo y el 10,0\% tienen BANA positivo.

Tabla 7. Comparación entre BANA antes y BANA después

\begin{tabular}{|c|c|c|c|c|c|c|c|}
\hline Bana & Numero & & & & & & \\
\hline Negativo & 0 & & & & & & \\
\hline Leve Positivo & 1 & & & & & & \\
\hline Positivo & 2 & & & & & & \\
\hline \multicolumn{8}{|c|}{ Estadísticas de muestras emparejadas } \\
\hline \multirow[b]{3}{*}{ Bana } & & $\mathbf{N}$ & Media & Diferencia & $\begin{array}{l}\text { Desviación } \\
\text { estándar }\end{array}$ & T student & Wilcoxon \\
\hline & Antes & 30 & 1,60 (Positivo) & \multirow[b]{2}{*}{$-0,830$} & 0,50 & \multirow[b]{2}{*}{0,000} & \multirow[b]{2}{*}{0,000} \\
\hline & Después & 30 & $\begin{array}{c}0,77 \\
\text { (Leve positivo) }\end{array}$ & & 0,63 & & \\
\hline
\end{tabular}

La media del BANA antes del tratamiento es de 1,6 que se aproxima a 2 , que equivale a positivo, con una desviación estándar de 0,5. La media del BANA después es de 0,77 que se aproxima a 1 que equivale a Leve positivo.

El BANA tiene una disminución significativa $(p<0,05$, tanto en pruebas paramétricas como en no paramétricas) entre el antes y después, cambia de Positivo a Leve positivo.

\section{DIScusióN}

En los resultados de este estudio nos indican que hay una correlación positiva el cual se manifiesta con un color azul intenso, entre la prueba BANA y los parámetros clínicos periodontales, indicando que la enfermedad se encontraba en un periodo de niveles más altos de inflamación y un riesgo severo periodontal. 
No obstante, cuando los pacientes se encontraron en la fase restablecimiento después del raspado y alisado radicular los índices de correlación fueron positivos, existiendo mayor cantidad de colonias bacterianas presentes.

El test BANA reconoce los productos enzimáticos de los microorganismos conocidos como periodontopatógenos el agravio de sustrato $\mathrm{N}$ - $\alpha$-bencil-DL-arginina-2-naftilamida, nos indica una coloración con el cual se puede reconocer la unidad formadora de colonias (UCF) bacterianas ${ }^{13}$. El procedimiento periodontal que se realizó, así como también otros análisis han probado que la terapia periodontal que incluyen el raspado y alisado radicular sea sólo o con mediadores coadyuvantes las cuales tienen acciones beneficiosas sobre los cambios del microbiota subgingival esperando como resultado un mejoramiento del estado clínico de los usuarios tratados ${ }^{14}$.

Existiendo una correlación positiva entre la profundidad de sondaje y los valores de la prueba BANA que se realizó en nuestra investigación, también se demostró que el trabajo realizado por Grisi, Novaes $^{15}$, encontrándose profundidades de 2 a $3 \mathrm{~mm}$ que indicaron valores positivos en los valores de la prueba BANA, asumiendo que incluso reducidos cambios gingivales ocasionados por la presencia de espiroquetas, pueden incidir en los resultados de la prueba. Cuando se observó el estudio realizado por Muthukumar, Anand ${ }^{16}$, realizo la valoración del sangrado gingival como un parámetro clínico para la evaluación de condiciones inflamatorias gingivales, demostrando que un gran porcentaje es decir el $80 \%$ de los sitios con sangrado gingival al sondaje, mostrando pruebas BANA débil positivo o positivo. Estos resultados son muy parecidos a los aplicados a nuestro estudio donde el $100 \%$ los sitios con cierto grado de inflamación en la base que aplican valores BANA positivo. Teniendo una semejanza con lo expuesto por este autor, y colaboradores donde indican como el sangrado al sondaje es una herramienta muy útil en el diagnostico periodontal y la evolución del tratamiento, a pesar del cierto grado de subjetividad que presenta ${ }^{17,18}$.

En cuanto al Nivel de Inserción, es el parámetro clínico que evidencio mayor correlación con la prueba BANA en esta labor de investigación. El nivel de inserción es un parámetro clínico muy fundamental ya que está asociado a la perdida de tejido de soporte del elemento dental, y que ha demostrado gran variabilidad entre diferentes poblaciones ${ }^{19}$

Se ha podido también encontrar a otros autores que evidenciaron cierta correlación de Nivel de Inserción indicado correlación con la prueba BANA ${ }^{20}$.

Dentro de las restricciones de este estudio podemos destacar la ausencia de los individuos en el grupo test lo que dificulta explorar nuestros resultados en el resto de la población, sin embargo los resultados encontrados concuerdan con otros trabajos previamente citados, así también se debe enfatizar que el corto tiempo la revaluación de los participantes no permite un reporte de los datos a largo plazo tanto los resultados así como la evaluación a larga data, los parámetros clínicos periodontales como los obtenidos por la prueba BANA.

\section{CONCLUSIONES}

- Se identificaron microorganismos a la prueba BANA y los resultados del estudio no mostraron diferencias significativas entre el grupo de pacientes antes y después de la terapia periodontal no quirúrgica.

- Se aplicaron la prueba con el Test BANA los resultados dieron POSITIVOS Y LEVES POSITIVOS, antes y después de la terapia periodontal.

- En cuanto a las correlaciones de los parámetros clínicos periodontales no variaron entre pacientes tratados y no tratados de acuerdo a la prueba BANA.

- Se compararon de los análisis microbiológicos del test BANA, dando como resultados que antes de la terapia no quirúrgica se obtuvo un BANA POSITIVO, y el resultado después de la terapia no quirúrgica se obtuvo un BANA LEVE POSITIVO, observando estadísticamente una disminución significativa.

- La media del BANA antes del tratamiento es de 1,6 que se aproxima a 2 que equivale a positivo, con una desviación estándar de 0.5.

- La media del BANA después es de 0,77 que se aproxima a 1 que equivale a Leve positivo.

- El BANA tiene una disminución significativa $(p<0,05$, tanto en pruebas paramétricas como en no paramétricas) entre el antes y después, cambia de positivo a Leve positivo.

\section{CONTRIBUCIÓN DE LOS AUTORES}

Juana Alexandra Ayo Bedón: Concepción y diseño del trabajo; recolección y obtención de resultados; análisis e interpretación de datos; redacción del manuscrito.

Mariela Cumandá Balseca Ibarra: Concepción y diseño del trabajo; recolección y obtención de resultados; análisis e interpretación de datos; redacción del manuscrito.

\section{CONFLICTO DE INTERESES}

Los autores declararon no tener ningún conflicto de interés personal, financiero, intelectual, económico 
y de interés corporativo con el Hospital Metropolitano y los miembros de la revista MetroCiencia.

\section{FINANCIAMIENTO}

El financiamiento para la realización de la presente investigación fue financiado por los autores.

\section{REFERENCIAS BIBLIOGRÁFICAS}

1. Friedewald VE, Kornman KS, Beck JD, Genco R. The American Journal of Cardiology and Journal of Periodontology Editors' Consensus: periodontitis and atherosclerotic cardiovascular disease. Journal of Periodontology. $2009 \mathrm{Jul}$; 80(7): p. 1021-32.

2. Arrieta Blanco JJ, Bartolomé Villar B, Jiménez Martinez E, Saavedra Vallejo P. Arrieta Blanco FJ. Problemas dentales en pacientes con diabetes mellitus (II): índice gingival y enfermedad periodontal. Medicina Oral. Pud Med. 2003 ene; 8(1).

3. Santos R, Foss M, Rocha G. Impact of periodontitis on the diabetes related infl ammatory status. Rev Estomatol Herediana. 2015 Oct; 25(3): p. 278-287.

4. Yamazaki K, Honda T, Oda T, Ueki-Maruyama K, Nakajima T. Effect of periodontal treatment on the $\mathrm{C}$-reactive protein and proinflammatory cytokine levels in Japanese periodontitis patients. J Periodontal Res. 2005 Feb; 40(1): p. 53-8

5. Löe H. Periodontal disease: The sixth complication of diabetes mellitus. Diabetes Care. 2011 Jun; 18(31)

6. Bascones Martínez A FRE. Periodontal diseases as bacterial infection. Av Periodon Implantol. 2005 Dic; 17(3): p. 111-118.

7. Newman MG. Periodontología Clínica de Carranza. 8th ed. México: AMOLCA; 2011.

8. Martínez Lizan MI, Manau Navarro C, Ramon Torrell JM, Cuenca Sala E, Echeverría García JJ. Efecto de un colutorio de fluoruro estañoso-fluoruro de aminas (Lemiro|@) sobre el índice de placa en ausencia de cepillado. Archivos de Odonto Estomatología. 1993; 9(8): p. 454-456.

9. Martins Chávarry NG, Vianna Vettore M, Carmelo S, Sheiham A. The relationship between diabetes mellitus and destructive periodontal disease: a meta-analysis. Oral Health Prev dent. 2009 Feb; 7(2): p.
107-27.

10. Grisi M, Correa F, Fanganiello C. Relación entre la presencia o ausencia de sangrado gingival y el test enzimático de BANA. Braz Dent J. 2001 Sep; 12(1): p. 23-26.

11. Ledesma M, Miñarro R, Gacés O, Martínez A. La reacción BANA como método de diagnóstico de periodontitis activa. ADM. 1995 Ene; 52(1): p. 254-258

12. GRISI MFdM, NOVAES AB, ITO IY, SALVADOR SLdS. Relationship between Clinical Probing Depth and Reactivity to the BANA Test of Samples of Subgingival Microbiota from Patients with Periodontitis. Braz Dent J. 1998; 9(2): p. 77-84.

13. Loesche W, Giordano P. The utility of the BANA test for monitoring anaerobic infections due to spirochetes (Treponema denticola) in periodontal disease. J Dent Res. 1990 Oct; 69(10): p. 1696-702.

14. Socransky SS, Haffajee AD, Teles R, Wennstrom JL, Lindhe J, Bogren A. Effect of periodontal therapy on the subgingival microbiota over a 2 -year monitoring period. I. Overall effect and kinetics of change. J Clin Periodontol. 2016 Ago 771-80; 40(8)

15. Grisi M, Novaes A, Ito I, Salvador S. Relación entre la profundidad del sondeo clínico y la reactividad a la prueba BANA de muestras de microbiota subgingival de pacientes con periodontitis. Revista Brasileña de Odontología. 1998 Ene; 9(1): p. 77-84.

16. S. Muthukumar MVA. Relación entre el sangrado gingival y la infección periodontal anaeróbica evaluada mediante el ensayo BANA (N-Benzoyl-DL-Arginine- $\beta$-Napthylamide). J Pharm Bioallied Sci. 2014 Julio; 6(570-573)

17. Lang N, Adler R, Nyman S. Ausencia de sangrado en el sondeo. Un indicador de estabilidad periodontal. Journal Clinic Periodontology. 1990 Nov; 10(1): p. 714-721.

18. Weinberg MA, Hassan $\mathrm{H}$. Bleeding on probing: what does it mean? Gen Dent. 2012 Jul; 60(4): p. 271-6.

19. Al Mugeiren O. Evaluación del estado periodontal entre los pacientes ambulatorios que asisten a clínicas dentales de universidades privadas en la ciudad de Riad, Arabia Saudit. Journal of International Oral Health. 2018 Ago; 10(2): p. 192-197.

20. Morita M, Wang H. Relación del nivel de sulfuro sulcular con la severidad de la enfermedad periodontal y la prueba BANA. Journal Periodontology. 2011 Ene; 71(1): p. 74-78. 